\title{
DEM Modeling of Crushable Grain Material under Different Loading Conditions
}

\author{
M. Amine Benmebarek ${ }^{1 *}$, Majid Movahedi Rad $^{1}$ \\ ${ }^{1}$ Department of Structural and Geotechnical Engineering, Faculty of Architecture, Civil Engineering and Transport Sciences, Széchenyi \\ István University, Egyetem tér 1, Győr 9026, Hungary \\ * Corresponding author, e-mail: benmebarek.mohamed.amine@hallgato.sze.hu
}

Received: 28 January 2021, Accepted: 10 April 2021, Published online: XXXxxxxx 2021

\begin{abstract}
This paper deals with the effect of contact conditions on the crushing mechanisms and the strength of granular materials. The computation of crushable grain material under different loading conditions is performed using 3D model of discrete element method (DEM). The crushable macro-grain is generated from a large number of identical spherical micro-grains which are connected according to the bonded particle model. First, the parameters of the proposed DEM model are calibrated to match the force-displacement curve obtained from Brazilian Tests performed on cylinders made of artificially crushable material. The damage profile right at the point when the force-displacement curve reaches its maximum is seen to replicate the same crack patterns observed in Brazilian test experiments. Then, parametric investigations are performed by varying the coordination number, the contact location distribution, and the contact area. The results show that these parameters play a significant role in determining the critical contact force and fracture mechanism of crushable particles compared to a traditional macro-grain crushing test. Increasing distribution and coordination number of the macro-grain increases particle strength when large area contact is permitted. However, for linear contact area, the effect of increasing coordination number on particle strength is marginal.
\end{abstract}

\section{Keywords}

grain breakage, micro-grain, macro-grain, DEM simulation, fragmentation patterns

\section{Introduction}

Crushing mechanisms in granular materials are important processes for many geotechnical engineering applications. Under high stresses, grain breakage alters the grain size distribution and is usually accompanied by a reduction of the permeability and an increase in compressibility of the grain skeleton [1-4]. These complex microscale processes play a critical role in the performance and serviceability of many engineering applications, such as embankments [5], rockfill dams [6, 7], railway tracks and geotechnical engineering [8]. The mechanisms of grain crushing are complex and depend closely on the stresses transmitted through particle contacts.

Previous experimental observations revealed that the macro-grain crushing of a grain assembly under compression is influenced by many factors, including, local microstructure defects, particle size and shape, coordination number of the macro-grain (number of contacts to the particle [9]), position of the contact points, loading conditions, duration, and boundary conditions [10-15].
Numerous authors have developed failure models that take into account the coordination number in the assembly of grains. Some authors have proposed failure criteria for tensile [16] and plane shear [17] fracture modes taking into consideration the effect of the coordination number. The high coordination number is known by its effect for increasing the strength of particles and of preventing crushing. Wang and Arson [18] have clarified this mechanism by the redistributing of the compressive forces concentrated at the contact points of the particles in a distributed pressure close to the hydrostatic pressure conditions.

Nevertheless, the effects of the contacts position and contacts area on the fragmentation patterns and strength of the particles have not been deeply investigated. Few works have focused on the effect of these parameters. Todisco et al. [19-20] designed an experimental device to study multi-contact crushing of sand or limestone particles. Using Weibull's statistics, they pointed out that an increase in the coordination number leads to a decrease 
in the probability of crushing. They showed that in addition to the coordination number, the nature of those contacts, controlled by particle morphology and mineralogy, also plays a significant role in determining the strength of a particle. They exhibited that compressed particles with higher coordination number have a lower probability of crushing than compressed particles between two diametrical opposite forces.

Salami et al. [10], using original experimental apparatus with multiple contact points with both surface and linear contacts, demonstrated that the coordination number and their positions play an important role in the fragmentation of an individual grain and that the critical contact force is highly dependent on the contact area (the area of the contact between the macro-grain and the loading plate).

Using drop weight crushing tests, Artoni et al. [21] showed a strong effect of contact orientation on particle strength as well as similar fracture patterns.

From the review of experimental works, it should be noted that the coordination number was restricted to grains with a few coordination number less than or equal to 6 for Salami et al. [10] and 4 for Artoni et al. [21]. This restriction is not representative of particles that are part of a well graded granular assembly. Further, it is difficult to define the stress and to capture critical contact force and crushing evolution in testing specimens. Compared with experimental investigations numerical simulations offer the chance to observe process dynamics in given time and spatial coordinates [21, 22]. Detailed micro and macro scale information can also be recorded. Reruns of the numerical simulations using different material and process parameters become possible for optimization. Significantly more information can be obtained from this model than by experiments. Thus, the use of numerical modeling seems to be unavoidable.

Many finite element, Lagrangian finite difference, and boundary element programs have interface elements or "slip lines" that allow them to model discontinuous material or crack propagation to some extent. However, their formulation is generally restricted in the cases of the use of numerous cross interfaces, total separation of the parts of the model or the need to recognize new contacts.

According to McDowell et al. [23], the discrete element method DEM, using the particle flow code (PFC3D), has been one among the foremost common numerical approaches to model fracture in granular materials. This approach provides a virtual laboratory to simulate mechanical tests without the limitations of the size and controlling particle multi-loading encountered in the laboratory. In this context, DEM offers an attractive alternative in that the bonded particles can be broken apart and without any assumption of a custom model. For these reasons, in this paper, the effect of different loading conditions on the fragmentation patterns and particle strength are examined using experimental Brazilian laboratory tests and discrete element method (DEM) model. The 3D crushable granular material is made up from a large number of micro grains which are connected according to the properties of crushable parallel bond. According to the experimental results, the proposed 3D discrete element method model is first calibrated. Then, parametric studies are carried out to study the effects of coordination number, location, and contact area. Finally, the results are compared with other investigations and discussed.

\section{Experiments: Brazilian strength test}

Systematic experimental testing of samples crushing for natural materials is usually difficult due to the relatively high stress required to crush the grains, and also the variability and heterogeneousness of natural deposits, which makes it difficult to get repetitive results. For these reasons, in order to obtain similar test results, an artificial material was considered for experimental Brazilian laboratory tests where cement and silt were mixed according to their mass ratio in which cement provides bonding and silt is the main filling material. Cylinder specimens with a diameter of $60 \mathrm{~mm}$ and height of $60 \mathrm{~mm}$ were tested using the Brazilian strength test in a static loading machine. Displacement transducer was used for the measurement. The vertical load was respectively transferred to specimen through plywood trips with a width of $10 \mathrm{~mm}$. The specimens were compressed between two diametral opposite loading strips as shown in Fig. 1.

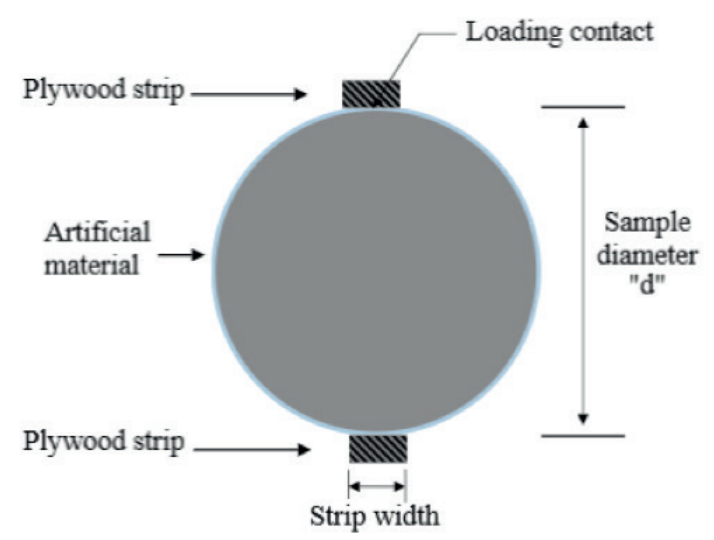

Fig. 1 Sketch of the initial geometry of the sample in the splitting tensile test 
During the rise of the externally applied load frame with a constant loading velocity of $v=0.025 \mathrm{~mm} / \mathrm{s}$, the reaction force was recorded as a function of displacement until the breakage of specimens. Thus, the load-displacement data has been plotted for all the specimens.

Several splitting tests on the artificial crushable specimens were carried out. In all tests the main vertical macro-crack first occurred at the mid-height of the specimen in the central vertical zone. Then it propagated towards the top and the bottom of the specimen orland branched to form a wedge directly under the plywood board. Finally, at failure, the concrete specimen was divided into two main parts as shown in Fig. 2(a).

Similar load-displacement results were measured for all Brazilian laboratory tests. Fig. 2(b) illustrates typical corrected load-displacement recorded for one representative of the samples. The correction includes the initial nonlinear displacement because of the loading contact defect and deformation of the plywood strip. A brittle failure was observed for all Brazilian tests. The applied force increases with increasing of the prescribed vertical

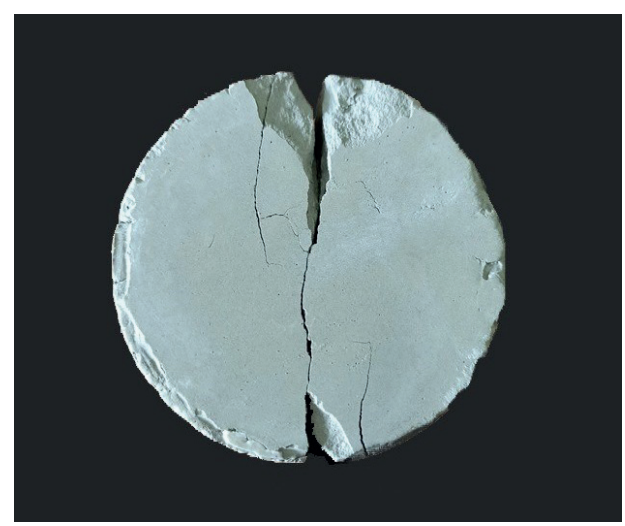

(a)

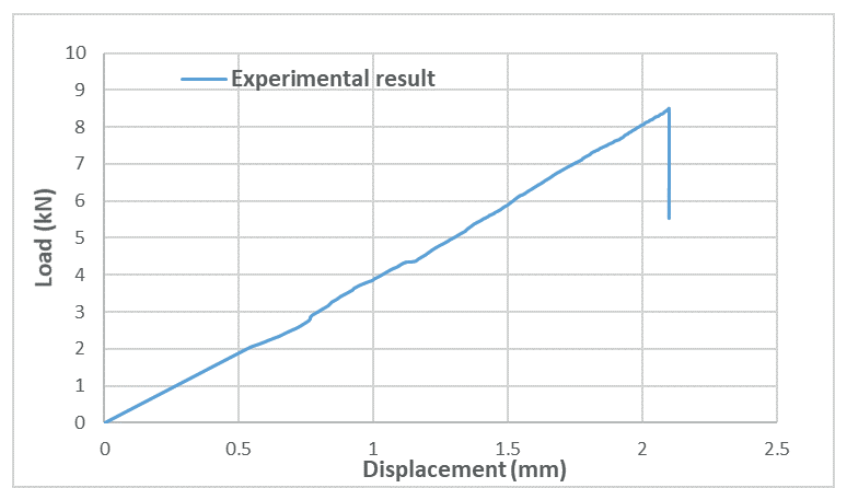

(b)

Fig. 2 (a) Typical failure observed for artificial cylinder samples subjected to Brazilian test; (b) Typical load-displacement diagram of artificial material in the Brazilian tests displacement and it drops suddenly after the peak point. The average peak contact load $8.4 \mathrm{kN}$ was reached at vertical diameter rapprochement of $2.2 \mathrm{~mm}$.

\section{Discrete numerical model}

\subsection{Contact models}

All numerical computations were carried out through the particle flow code PFC3D [24]. With PFC3D code it is possible to reproduce many behaviors observed in solid materials by using contact model that allows bonds at contacts to be created. In the bonded balls implemented in PFC3D, the linear parallel bond consists of a linear model and a parallel bond interfaces. The linear model interface can carry only a non-tensional force and slipping in the shear (tangential) direction is governed by a Coulomb criterion. Parallel bond interfaces mimic the role of a sticky grain cementing agent that can transmit forces and moments between the bounded particles. Therefore, bonds conceptually represent material between pieces (i.e., balls, and facets) that may carry loads to specified limits. During computation, if contact stresses are below strength limits, the material can act in a similar way to a continuum approximation of a solid. Once the tensile strength or shear strength of a bond is reached during loading, bonds break and neighboring pieces are free to behave like they are in an un-bonded state. For instance, frictional slip may occur locally, simulating the initiation and propagation of fractures. The bonded-particle modeling methodology is discussed thoroughly in Potyondy [25]. It should be noted that the linear parallel bond contact model in PFC produces unrealistically low ratios of compressive to tensile strength (Potyondy, [25]). To overcome this shortcoming, it is more suitable to use the flat joint contact model. However, assigned the same properties, flat joint contact and parallel bonded models give almost the same tensile strength (Itasca Consulting Group [24]).

For this analysis, two types of contacts will be present: ball-ball and ball-facet contacts. The linear contact model was used for the contact between balls of macro-grain and facets of loading system. However, the linear parallel bond model was adopted for ball-ball to generate a bonded macro-grain.

\subsection{DEM Model calibration}

For the DEM model proposed for simulation of the behavior of the tested artificial crushable material, the crushable macro-grains have a cylinder shape with a diameter and height of $60 \mathrm{~mm}$ and generated using non-breakable micrograins "balls". With the aim of reproducing the conditions 
of the experiment, the diameter of the micro-grains was set to $3 \mathrm{~mm}$. The loading platens were modeled by plate rigid walls. First identical balls were generated, confined, and bonded in the form of cylindrical macro-grain. Then, stiffness and bond parameters of the DEM cylindrical macro-grain model were calibrated to better matching forcedisplacement curves obtained with our experiments during Brazilian crushing tests performed on cemented silt.

To simulate the initial state before loading, first, the randomly generated balls created using the ball distribute command were confined in a closed material vessel representing the geometry of the macro-grain. The linear bond model for ball-ball and ball-facet contacts was selected. After computing equilibrium, this state was accompanied by an initial overlap between balls. The model cycle command was given with the calm keyword to periodically null all ball translational/rotational velocities, effectively removing kinetic energy from the system. This allows the balls to be rearranged without significant overlap and without significant movement, achieving a dense packing relatively rapidly. Then, we installed parallel bond to ballball contacts and removed the vessel wall to obtain initial arrangement before loading, due to the overlap, some of the loads were transferred to stretching the parallel bonds. To observe this transfer, a measurement sphere can be created, and history monitoring can be set up to record the evolution of the stresses within the assembly. With a high overlap, the assembly specimen explodes. To remove this perturbation, one must reset the contact forces contact moments as well that have been accumulated to the bodies during the previous force-displacement calculation cycle.

In the early stage of calibration, the Young's modulus of the particle $E^{*}$, Young's modulus of the parallel bond, $\bar{E}$, normal-to-shear stiffness ratio $K^{*}=K_{n} / K_{s}$ and the ratio of normal to shear stiffness of the parallel bond, $\bar{K}_{n} / \bar{K}_{s}$ were chosen to match the linear response, elastic part, before the peak load. The grain and cement moduli and ratios of normal to shear stiffness were set equal to one another to reduce the number of free parameters. The frictional coefficient, which is known to not greatly influence DEM results (Potyondy and Cundall) [26], was set to 0.5 as recommended in the state of the art (Wang et al.) [27]. The linear behavior was found to be highly governed by Young's modulus.

The second stage of calibration involved a series of numerical tests to match the peak load. The tensile bond strength and the shear strength of the parallel bond were strictly linked to the peak load and crush pattern (Itasca, PFC). The tensile strength was chosen in accordance with the peak load and the shear strength was chosen arbitrary greater than tensile strength to permit both tensile crack and shear crack to develop during loading.

The calibrated parameters are shown in Table 1. It should be pointed out here that as the study in this paper focuses mainly on the different loading conditions on the fragmentation patterns and particle strength some parameters were chosen simply.

The response of a DEM model is sensitive to the loading rate which must be slow enough to ensure a quasi-static response. Therefore, a loading velocity sensitivity analysis was performed under different wall velocities with the control of peak loading force and the ratio of the unbalanced force magnitude to the applied force magnitude. Considering the quasi-static condition and computational efficiency, the selected loading velocity applied to the loading platform was $0.01 \mathrm{~m} / \mathrm{s}$ generating an unbalanced force to contact force ratio of less than 0.001 during the crushing process. By further reducing the velocity, the results showed no effect on the peak load however the computation time became troublesome.

During the entire simulation, the contact force, as well as the number of broken bonds were recorded.

Typical simulation results of calibrated model are shown in Fig. 3, in which we can see that once the peak force is reached, the macro-grain breaks into two main parts (Fig. 4), which is in agreement with experimental observations (Fig. 2(a)). The macro-grain was randomly rotated and tested where it gave similar results which confirm that there is no weak area inside the macro-grain as well as crystal-like groups often observed in two-dimensional simulation.

Table 1 Parameters used in the DEM simulation

\begin{tabular}{lcc}
\hline Elementary particle size, $D$ & $\mathrm{~mm}$ & 3 \\
Installation gap, g ${ }^{-} \mathrm{mm}$ & 0.002 \\
Density & $\mathrm{kg} / \mathrm{m}^{3}$ & 2400 \\
Young's modulus of the particle, $E^{*}$ & $\mathrm{GPa}$ & 0.14 \\
$\begin{array}{l}\text { Ratio of normal to shear stiffness of the } \\
\text { particle, } K_{n} / K_{s}\end{array}$ & 1 \\
$\begin{array}{l}\text { Particle friction coefficient, } \mu \\
\text { Young's modulus of the parallel bond, } \bar{E}\end{array}$ & $\mathrm{GPa}$ & 0.14 \\
$\begin{array}{l}\text { Ratio of normal to shear stiffness of the } \\
\text { parallel bond, } \bar{K}_{n} / \bar{K}_{s}\end{array}$ & & 1 \\
$\begin{array}{l}\text { Tensile bond strength } \\
\text { Cohesion bond strength }\end{array}$ & $\mathrm{MPa}$ & 1.3 \\
\hline
\end{tabular}




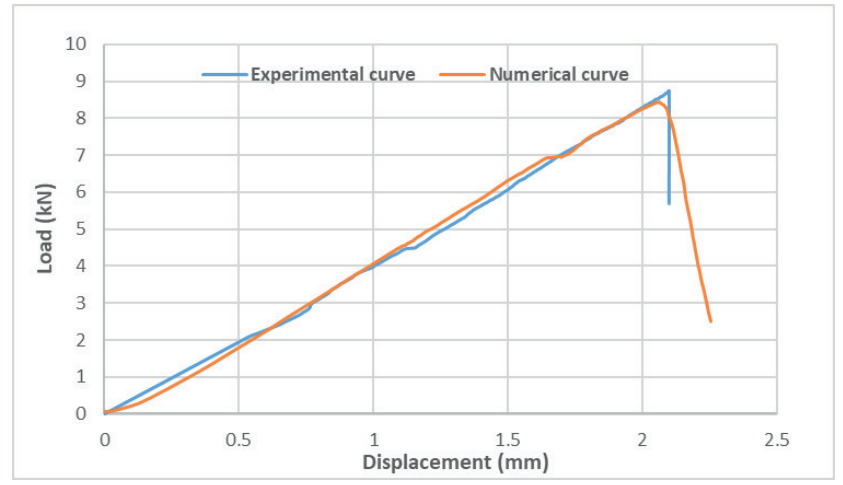

Fig. 3 Load-displacement curve during the Brazilian test simulated by the DEM: calibration of DEM macro-grain model against experimental test results

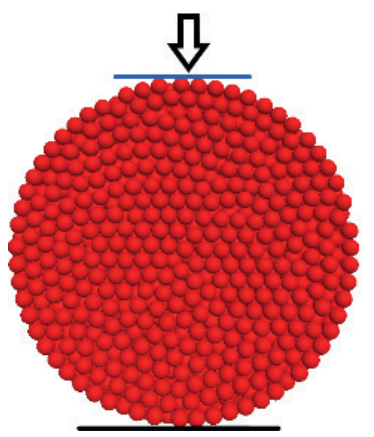

(a)

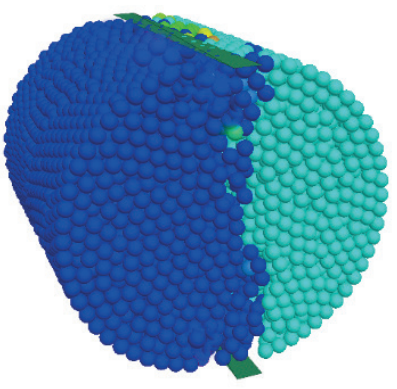

(b)
Fig. 4 Fracture patterns for calibrated model: (a) initial state; (b) at the peak load

\section{Results of computation and discussion}

The strength and breakage of bonds in a crushable macro-grain were examined for different coordination number, contact location and type of contact. These tests were chosen to mimic true grain conditions in granular materials.

Fig. 5 sketches the geometry examined for different coordination number and location. The macro-grain is investigated for diametric contact, three contacts with different angles $\alpha$, four contacts with 90 degree and eight contacts with 45 degree.

Three contact models were considered plate contact model, cylinder contact model and linear contact model. These three models are outlined in more detail in the following.

\subsection{Effect of contact area for diametral compression}

The effect of contact area for diametral compression test was examined for plate, cylinder and linear contacts covering the majority of the natural contacts in reality between the grain assembly. Within the loading phase, both the top and bottom walls were under a constant velocity.

Fig. 6 shows the captured fracture patterns for these three types of contact area. The fracture patterns are similar to that obtained experimentally in Brazilian test.
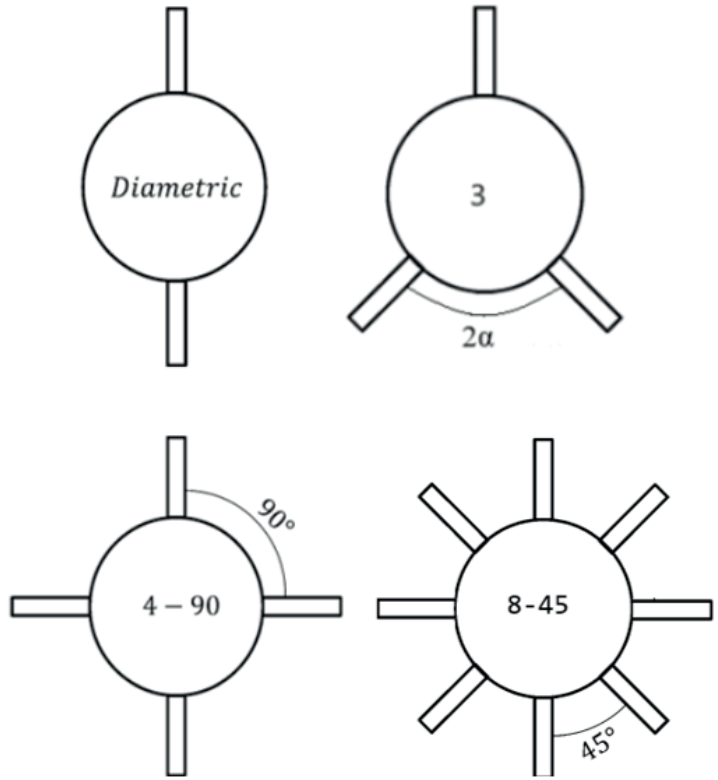

Fig. 5 Sketch of the geometry examined for different coordination number and location

A diametric plane fracture is found, which is initiated by the breakage of a few bonds close to the vertical diametric plane. Then, bonds continue to progressively break around the vertical diametric plane until the crack relates the two loading points. Force-displacement curves obtained from simulations are plotted in Fig. 7. For all contact types, the loading force first increases substantially linear with the increasing displacement of the wall till it reaches the peak point where it drops sharply. From the force displacement diagram the highest peak loading of the crushable macro-grain is firstly the case of plate contact $8.4 \mathrm{kN}$, second the case of cylinder contact with a peak load of $7.5 \mathrm{kN}$, then the case of linear contact with peak load of $3.4 \mathrm{kN}$. So, we can say that the peak loading and the apparent stiffness of the macro-grain in case of plate contact are greater than cylinder and linear contact.

The high peak load discrepancy between surface contact and linear contact might be attributed to the higher concentration of forces near the contacts in the case of a contact along a line. These numerical tests demonstrate the strong effect of surface contact in diametric loading tests.

The commonly used indirect tensile strength of a cylindrical sample with diameter $\mathrm{D}$ and thickness $\mathrm{T}$ is given by:

$\sigma=\frac{2 P_{\max }}{\pi D T}$

where $P_{\max }$ is the peak load and $D$ and $T$ are the diameter and the thickness of the specimen respectively. 
This expression gives $P_{\max }=7.35 \mathrm{kN}$ which is close to the results of plate and cylinder contacts. Comparing the peak load $P_{\max }$ predicted by Eq. (1) with the present results shows good agreement with the cases of plate and cylinder contacts however high discrepancy is noted with the case of linear contact.

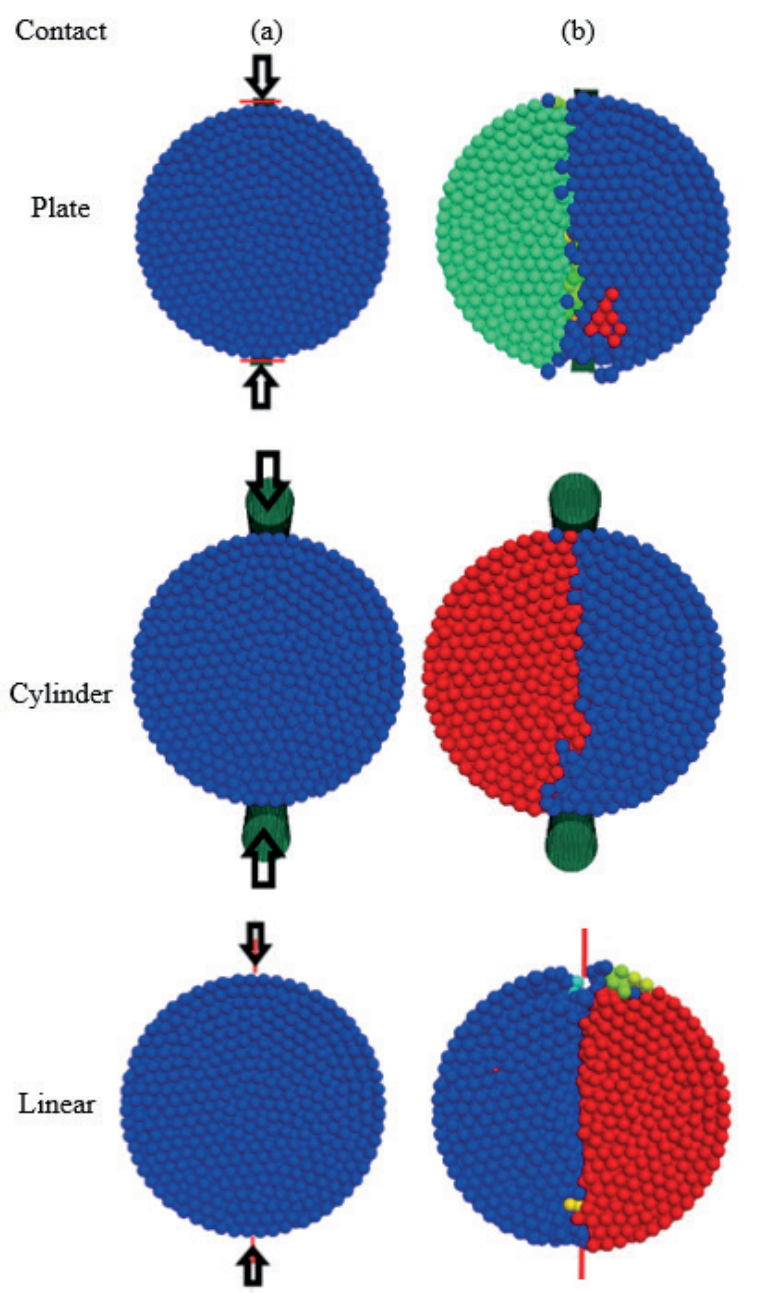

Fig. 6 Fracture patterns for diametral cases: (a) initial state; (b) at the peak load

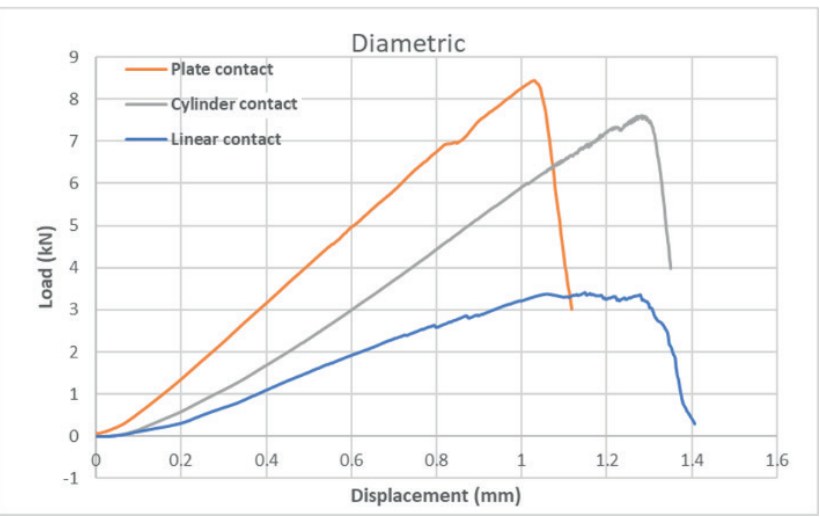

Fig. 7 Load-displacement curve for diametral cases

In addition, it should be noted that contact area strongly impacts the stiffness of the macro-grain. Fig. 7 shows clearly the decrease of the apparent stiffness with the decrease of loading contact area.

\subsection{Effect of contact area for four contacts: 4-90 configuration}

The effect of the plate, cylinder, and linear contact surface for the case of four contacts with 90 degree (4-90 configuration) was also investigated. Fig. 8 shows the fragmentation pattern. The macro-grain breaks into different parts ranging between 3 to 4 main parts. The results of forcedisplacement curve are plotted in Fig. 9. The results show a more complicated post-peak behavior, which is explained due to several major failures within the macro-grain.

The recording of loading-displacement curve shows that the peak load of the four contact cases increased compared to diametric loading cases. The peak load increase is more pronounced with the increase of contact area. For instance, peak load increases for plate contact and linear contact are $61 \%$ and $13 \%$ respectively. This larger load

Contact

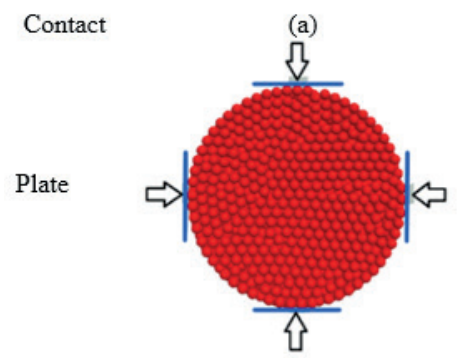

Plate
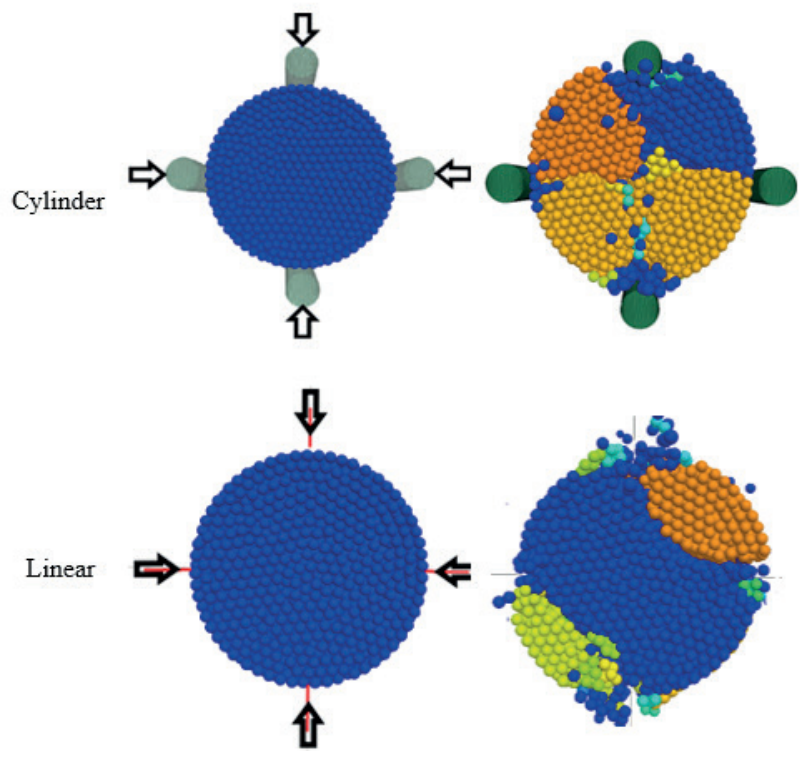

Fig. 8 Fracture patterns for 4-90 cases (a) initial state; (b) at the peak

load

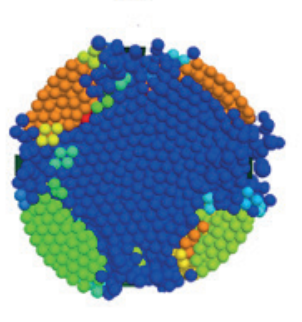

(b)

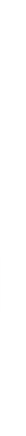




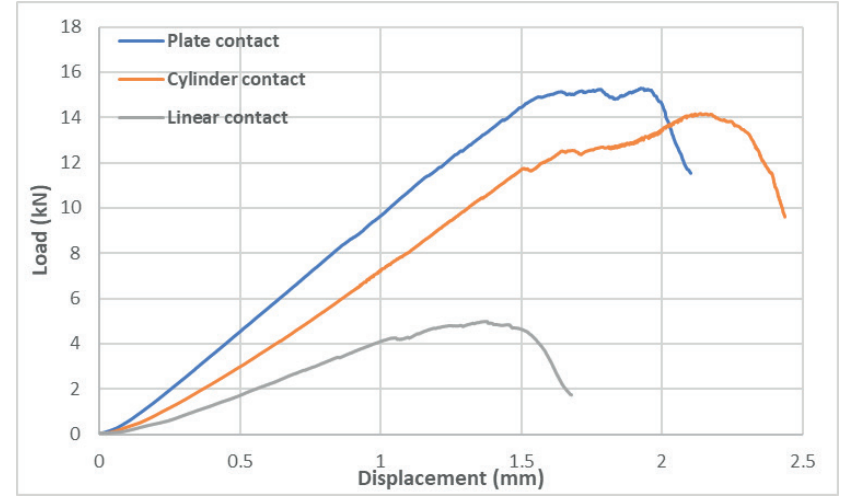

Fig. 9 Load-displacement curve for 90-4 cases

can be credited to the macro-grain breaks to more fragments in the four contacts case which infers more breakage inside the macro-grain compared to the diametric case and that leads to a larger loading breakage.

There was an importance difference between the current work and Salami et al. [10]. The computation results are in disagreement with those of Salami et al. [10] in which there was a presence of diametral crack and two secondary cracks between the top and lateral contacts. To explain these differences, focusing on the stresses applied on the sample Salami et al. [10] loaded the sample by giving the top plate a constant velocity, however lateral contacts were just put in contact with the specimen. Therefore, the lateral contacts were then passive. In contrast to our work, all the loading platen were moving towards the center.

\subsection{Three contacts: Effect of the location}

\subsubsection{Plate contact}

In order to examine the variation of contact locations, five configurations corresponding to five values of angle $\alpha$ (0: explained earlier in diametral compression, $20^{\circ}, 30^{\circ}$, $45^{\circ}$ and $60^{\circ}$ ) were examined. The two bottom walls were positioned symmetrically with their normal pointing towards the centerline of the cylinder.

Fig. 10 depicts the fragmentation pattern for $\alpha\left(20^{\circ}, 30^{\circ}\right.$, $45^{\circ}$ and $60^{\circ}$ ). The macro-grain breaks into 2,3 and 4 main different parts for $\alpha$ equal $0^{\circ}-20^{\circ}, 30-45^{\circ}$ and $60^{\circ}$ respectively. The essential results of the peak load, displacement at peak point and number of fragments for the case of plate contact are summarized in Table 2. In accordance with experimental and numerical results of Artoni et al. [21] it is clearly shown that the peak load increases with the rise of the angle $\alpha$. Compared to diametric loading where break in tension is dominant, this increase can be clarified by the mobilization of the friction contribution that
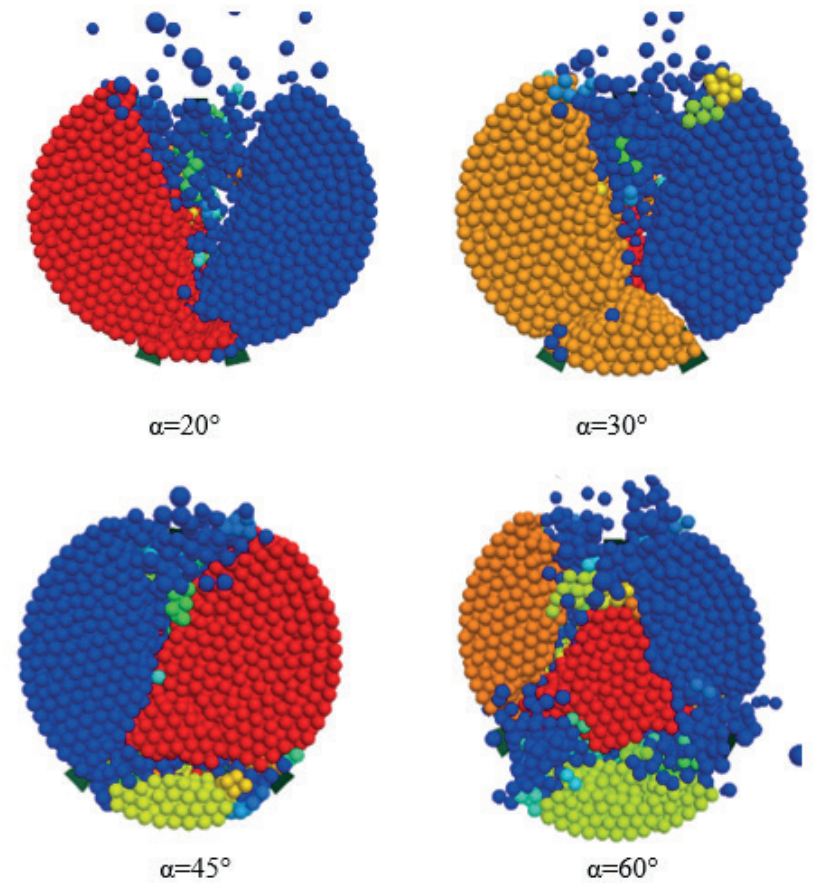

Fig. 10 Fracture patterns at the peak load for $\alpha$ plate contact cases

Table 2 results for different angle configuration: plate contact

\begin{tabular}{lccc}
\hline Contact & Peak load (kN) & $\begin{array}{c}\text { Displacement at } \\
\text { peak point (mm) }\end{array}$ & $\begin{array}{c}\text { Number of } \\
\text { fragments }\end{array}$ \\
\hline$\alpha=0^{\circ}$ & 8.4 & 1.05 & 2 \\
$\alpha=20^{\circ}$ & 9.35 & 0.85 & 2 \\
$\alpha=30^{\circ}$ & 9.98 & 1.02 & 3 \\
$\alpha=45^{\circ}$ & 10.9 & 1.25 & 3 \\
$\alpha=60^{\circ}$ & 11.6 & 1.49 & 4 \\
\hline
\end{tabular}

results to an increase in the shear component of the loading. As expected, and in accordance with previous work mentioned in the literature $[10,19-21]$ the position of contacts influences the peak load supported by the particle and therefore its apparent strength.

\subsubsection{Linear contact}

The effect of linear contact for five values of angle $\alpha$ $\left(20^{\circ}, 30^{\circ}, 45^{\circ}\right.$ and $\left.60^{\circ}\right)$ on the fragmentation patterns is shown in Fig. 11. For $\alpha$ ranging from $0^{\circ}$ to $30^{\circ}$, a diametral crack is observed. However, for $\alpha$ starting from $45^{\circ}$ to $60^{\circ}$ cracks oriented towards the centerline of the cylinder are noted. The results show that area contact can have a strong impact on the loading capacity and therefore the fragmentation patterns. Important results are summarized in Table 3. Contrarily to plate contact, the results of peak load show marginal effect of varying loading position and increasing coordination number. 

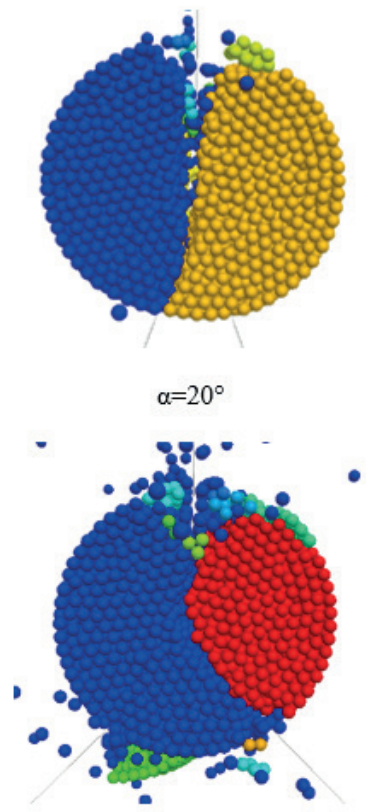

$\alpha=45^{\circ}$

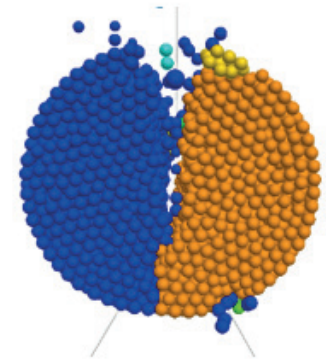

$\alpha=30^{\circ}$

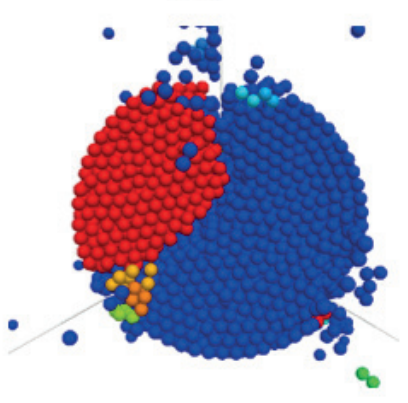

$\alpha=60^{\circ}$

Fig. 11 Fracture patterns at the peak load for $\alpha$ linear contact cases

Table 3 Results for different angle configuration: linear contact

\begin{tabular}{lccc}
\hline Contact & $\begin{array}{c}\text { Peak load } \\
(\mathrm{kN})\end{array}$ & $\begin{array}{r}\text { Displacement at } \\
\text { peak point }(\mathrm{mm})\end{array}$ & $\begin{array}{c}\text { Number of } \\
\text { fragments }\end{array}$ \\
\hline$\alpha=0^{\circ}$ & 3.34 & 1.09 & 2 \\
$\alpha=20^{\circ}$ & 3.45 & 0.97 & 2 \\
$\alpha=30^{\circ}$ & 3.74 & 0.99 & 2 \\
$\alpha=45^{\circ}$ & 3.72 & 1.07 & 2 \\
$\alpha=60^{\circ}$ & 3.62 & 1.05 & 2 \\
\hline
\end{tabular}

\subsection{Coordination number}

For this part, the increase of coordination number was examined for plate contact cases. Fig. 12 displays a representation of fracture patterns and broken bonds obtained for four coordination number cases: 2, 3, 4 and 8. As mentioned above, for two contacts case a vertical crack is formed. For three contact edges, three cracks are obtained between the top wall and the lateral contact walls. The cracks start at within the upper part of the sample and then spread towards the side walls and form a triangular crack.

For the four contact edges, a cloud of broken bonds starts accumulating near the platen walls with some minor broken bonds spreading inside the body of the sample. When the peak load is reached the form of square crack is hardly seen. For the eight contacts, the broken bonds appear to form a cloud around the sample and shape a circle. After the peak load, a large number of small fragments occurs around the body surface while the core of sample remains together. There was no obvious path for the fracture pattern in this case, but deterioration of the sample periphery is evident.
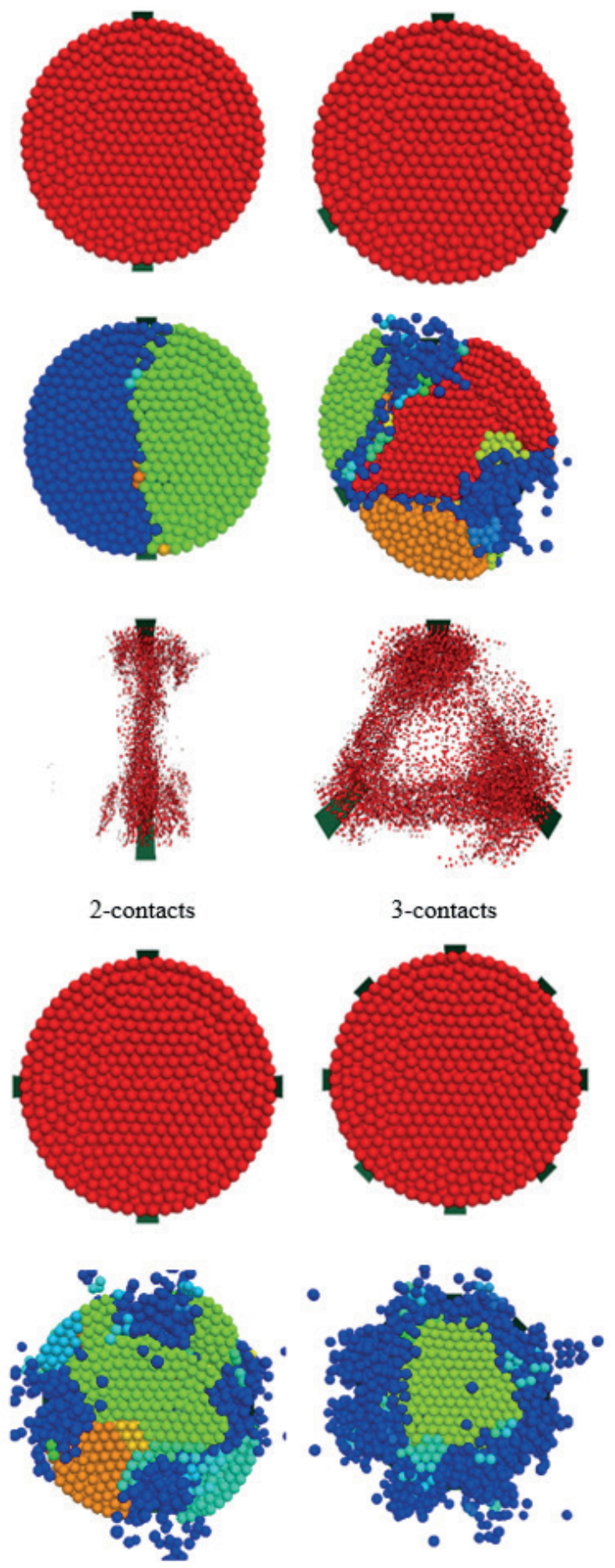

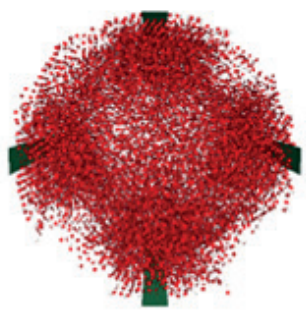

4-contacts

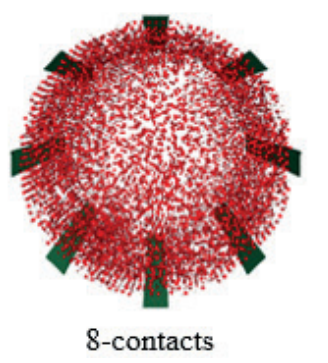

Fig. 12 Fracture patterns for different contact number 
Fig. 13 presents the results of force-displacement for these cases. From the force displacement curve, the force is increasing linearly with the same slope. The peak load for the case of 8 contacts is $16.7 \mathrm{kN}$ which represents an increase of $100 \%$ compared to the case of diametric contact $(8.3 \mathrm{kN})$. Therefore, the current computations confirm that a large number of contacts prevents the particles from crushing and are in good accordance with the experimental work conducted by Todisco et al. [19] where they confirmed that a higher contact number resulted in a decreased probability of crushing.

In addition, it was noted that the increase of the coordination numbers with isotropic distribution affects the fracture patterns leading to non-fragility splitting failure. The obtained failures correspond more to shear fracture mode II according to Ben-Nun and Einav [28]. For the cases of 4-contacts and 8-contacts there is no crack concentration in the center of the sample due to the confinement effect of loading (Fig. 12), and shear and tensile micro-cracks are concentrated at the particle periphery. After peak load, contact spheres broken bond by shear exhibit friction resistance leading to non-fragility splitting failure.

In Tsoungui's criterion, the failure is based on the analytical elastic solution of the distribution of stress in a disc grain subjected to two opposite forces. The tensile stress $\sigma_{x x}^{0}$ acting at the disc center is expressed by

$\sigma_{x x}^{0} \approx \frac{F}{\pi R}$

where $F$ is the external force and $R$ is the radius of the disc.

When a grain is subjected to an arbitrary set of forces, the complex distribution of forces is replaced by an equivalent distribution (see Fig. 14(b)) of orthogonal forces $F_{\text {max }}$ and $F_{\min }$. Then, $\sigma_{x x}^{0}$ expressed by Eq. (3) for equivalent set of contact forces.

$\sigma_{x x}^{0}=\frac{F_{\min }-3 F_{\max }}{\pi R}$

The fracture occurs according to a tensile splitting mode I, in a manner similar to that of a grain between two diametrically opposed forces.

In this case, the crack originates when the tensile stress, $\sigma_{x x}^{0}$, overcomes $\sigma_{c}$, which is assumed to be equal to that of a grain compressed diametrically.

By expressing the tensile stress $\sigma_{x x}^{0}$, as a function of the hydrostatic stresses $\mathrm{p}$ and of the shear $\tau$, the failure criterion becomes:

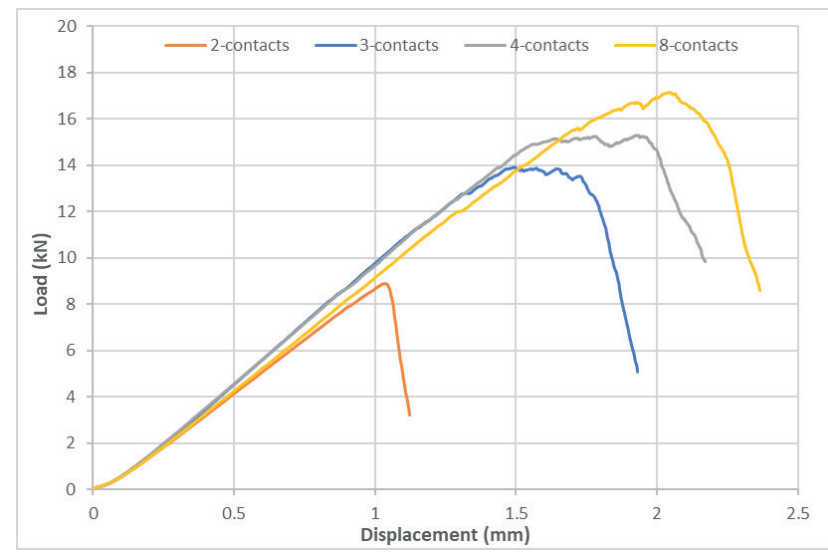

Fig. 13 Load-displacement curve for different contact number

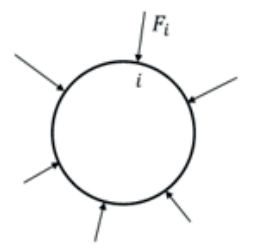

(a)

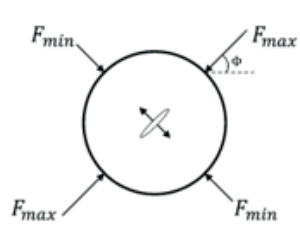

(b)

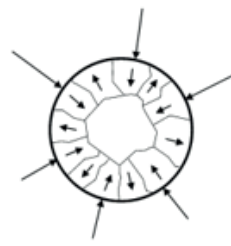

(c)
Fig. 14 Schematic of the two fracture criteria: (a) reference case; (b) opening fracture mode I according to Tsoungui et al. [16]; and (c) and in-plane shear fracture mode II according to Ben-Nun and Einav [28]

$\sigma_{x x}^{0}=2 \tau-p \geq \sigma_{c}$

From this relation, it is clear that this failure criteria is independent from the coordination number and loading conditions. Further, it can be inferred that no possible of breakage when the grain is subjected to isotropic loading configurations. This failure mode I is in accordance with the computation results only for or near diametral loading conditions. However, it is contradicted by the ruptures obtained numerically for isotropic loading as 3, 4 and 8 number of contacts with different contact area.

More than 2 number of contacts distributed uniformly around the macro-grain, the obtained failure mechanisms are in accordance with the second failure criteria, mode II, developed by Ben-Nun and Einav [28], for plane shear fracture modes taking into account the effect of the coordination number. According to the numerical study by Sukumaran et al. [29], particles under isotropic configuration will crush when the following critical force magnitude is met /overcome

$F_{c r i t}=d \sigma_{c} f_{w} f_{D} f_{C N}$,

where $\sigma_{c}$ is the tensile stress at failure of a grain of size $d$ and $f_{w}, f_{D}, f_{C N}$ are reduction factors taking account particle imperfections, particle curvature and coordination number 
respectively. The first factor $f_{w}$ is deduced from a statistical law [30] describing the distribution of the flaws or imperfections directly affecting the strength. The two other factors are defined empirically.

Sukumaran et al. [29] suggested capturing the curvature effect by the following partial factor:

$$
f_{D}=\frac{D / d}{D / d+1} \text {. }
$$

If the grain under question contacts a wall, we use $D \rightarrow \infty$, i.e., in which case this factor is omitted since $f_{D} \rightarrow 1$.

Further, Sukumaran et al. [29] proposed for any coordination number $C$ greater than 1 the following empirical relation.

$f_{C N}=(C-1) e^{\left(\frac{D}{d}\right)\left(\frac{(C-2)(C-3)}{4 C}\right)}$,

where $C$ is the coordination number of the crushing grain.

This expression satisfies the lower boundary of $f_{C N}=1$ when $C=2$ and suggests that as $C$ increases, the factor $f_{C N}$ increases, therefore, the critical force (Eq. (5)) grows, such that it is harder to crush the particle.

Nevertheless, the use of the critical force described by Eq. (5) for the present studied cases suffers in several ways. For plate and cylinder contacts where $D \rightarrow \infty$ implies $f_{D} \rightarrow 1, f_{C N} \rightarrow 1$ and. In addition, in the case of linear contact $D \rightarrow 0, f_{D} \rightarrow 0, f_{C N} \rightarrow(C-1)$ and $F_{\text {crit }} \rightarrow 0$.

\section{Conclusions}

To examine the impact of different grain loading conditions, the effects of contact number, location and contact area were investigated using experimental Brazilian Tests conducted on cylinders made of artificially crushable

\section{References}

[1] Alikarami, R., Andò, E., Gkiousas-Kapnisis, M., Torabi, A., Viggiani, G. "Strain localisation and grain breakage in sand under shearing at high mean stress: insights from in situ X-ray tomography", Acta Geotechnica, 10(1), pp. 15-30, 2014. https://doi.org/10.1007/s11440-014-0364-6

[2] Zhang, Y. D., Buscarnera, G. "A rate-dependent breakage model based on the kinetics of crack growth at the grain scale", Géotechnique, 67(11), pp. 953-967, 2017. https://doi.org/110.1680/jgeot.16.p.181

[3] Zhang, Y., Buscarnera, G. "Breakage mechanics for granular materials in surface-reactive environments", Journal of the Mechanics and Physics of Solids, 112, pp. 89-108, 2018. https://doi.org/10.1016/j.jmps.2017.11.008 material and numerical modeling using DEM PFC3D code. At first, a simple Brazilian laboratory tests performed on artificial crushable material was conducted to highlight diametral fracture pattern and the breakage strength. The DEM model parameters were then calibrated to better match the force-displacement results. Next, the calibrated DEM model was used to simulate the impact of various contact number, location and contact area. Distinct crack patterns were observed, connecting the loading contact to the other.

- For contact area, the results show that the critical force is highly dependent on the contact area. An increase in the contact surface leads to greater particle strength.

- The outcoming confirm that the strength of the particles increases with the number of contacts for the type of contact surface (i.e.: plate, cylinder). However, for linear contact, increasing the number of contacts is ineffective.

- In accordance with experiments reported by Artoni et al. [21], simulations predict a strong effect of the contact location on the particle strength and fracture patterns. Particle strength is higher when contacts are well distributed around particle.

- Fracture patterns are found affected strongly by contact number, contact location, and contact area. Three typical types of fracture are captured: two parts with a diametrical crack, three parts with cracks oriented to the center, four cracks connecting to contact points of loading and crack of particle periphery for higher number of contacts. For diametric compression, a central crack develops along the axis of diametral compression despite the type of contact.

[4] Chaudry, M. A., Wriggers, P. "On the computational aspects of comminution in discrete element method", Computational Particle Mechanics, 5(2), pp. 175-189, 2017. https://doi.org/10.1007/s40571-017-0161-8

[5] Kim, B., Prezzi, M., Salgado, R. "Geotechnical Properties of Fly and Bottom Ash Mixtures for Use in Highway Embankments", Journal of Geotechnical and Geoenvironmental Engineering, 131(7), pp. 914-924, 2005. https://doi.org/10.1061/(asce)1090-0241(2005)131:7(914)

[6] Zhou, W., Li, S., Ma, G., Chang, X., Ma, X., Zhang, C. "Parameters inversion of high central core rockfill dams based on a novel genetic algorithm", Science China Technological Sciences, 59(5), pp. 783-794, 2016 https://doi.org/10.1007/s11431-016-6017-2 
[7] Kermani, M., Konrad, J.-M., Smith, M. "In Situ Short-Term and Long-Term Rockfill Compressibility as a Function of Void Ratio and Strength of Parent Rock", Journal of Geotechnical and Geoenvironmental Engineering, 144(4), Article No. 04018009, 2018.

https://doi.org/10.1061/(asce)gt.1943-5606.0001835

[8] Indraratna, B., Sun, Y., Nimbalkar, S. "Laboratory Assessment of the Role of Particle Size Distribution on the Deformation and Degradation of Ballast under Cyclic Loading", Journal of Geotechnical and Geoenvironmental Engineering, 142(7), Article No. 04016016, 2016.

https://doi.org/10.1061/(asce)gt.1943-5606.0001463

[9] Jouannot-Chesney, P., Jernot, J. P., Cristian, L. "Pratical determination of the coordination number in granular media", Image Analysis \& Stereology, 25(1), pp. 55-61, 2006

https://doi.org/10.5566/ias.v25.p55-61

[10] Salami, Y., Dano, C., Hicher, P.-Y. "An experimental study on the influence of the coordination number on grain crushing", European Journal of Environmental and Civil Engineering, 23(3), pp. 432448, 2019.

https://doi.org/10.1080/19648189.2017.1285251

[11] Zhang, Y. D., Buscarnera, G., Einav, I. "Grain size dependence of yielding in granular soils interpreted using fracture mechanics, breakage mechanics and Weibull statistics", Géotechnique, 66(2), pp. 149-160, 2016.

https://doi.org/10.1680/jgeot.15.p.119

[12] Zhou, M., Song, E. "A random virtual crack DEM model for creep behavior of rockfill based on the subcritical crack propagation theory", Acta Geotechnica, 11, pp. 827-847, 2016. https://doi.org/10.1007\%2Fs11440-016-0446-8

[13] Sohn, C., Zhang, Y. D., Cil, M., Buscarnera, G. "Experimental assessment of continuum breakage models accounting for mechanical interactions at particle contacts", Granular Matter, 19(4), Article No. $67,2017$.

https://doi.org/10.1007/s10035-017-0750-5

[14] Ovalle, C., Frossard, E., Dano, C., Hu, W., Maiolino, S., Hicher, P.-Y. "The effect of size on the strength of coarse rock aggregates and large rockfill samples through experimental data", Acta Mechanica, 225(8), pp. 2199-2216, 2014. https://doi.org/10.1007/s00707-014-1127-z

[15] Cavarretta, I., O'sullivan, C. "The mechanics of rigid irregular particles subject to uniaxial compression", Géotechnique, 62(8), pp. 681-692, 2012.

https://doi.org/10.1680/geot.10.p.102

[16] Tsoungui, O., Vallet, D., Charmet, J.-C. "Numerical model of crushing of grains inside two-dimensional granular materials", Powder Technology, 105(1-3), pp. 190-198, 1999. https://doi.org/10.1016/s0032-5910(99)00137-0

[17] Ben-Nun, O., Einav, I. "A Refined DEM Study of Grain Size Reduction in Uniaxial Compression", In: The 12th International Conference of International Association for Computer Methods and Advances in Geomechanics (IACMAG), Goa, India, 2008, pp. 702-708
[18] Wang, P., Arson, C. "Discrete element modeling of shielding and size effects during single particle crushing", Computers and Geotechnics, 78, pp. 227-236, 2016.

https://doi.org/10.1016/j.compgeo.2016.04.003

[19] Todisco, M. C., Wang, W., Coop, M. R., Senetakis, K. "Multiple contact compression tests on sand particles", Soils and Foundations, 57(1), pp. 126-140, 2017.

https://doi.org/10.1016/j.sandf.2017.01.009

[20] Todisco, M., Coop, M., Guo, Q., Senetakis, K. "The effect of the coordination number on particle crushing", In: Geomechanics from Micro to Macro, Cambridge, UK, 2014, pp. 1063-1068. https://doi.org/10.1201/b17395-191

[21] Artoni, R., Neveu, A., Descantes, Y., Richard, P. "Effect of contact location on the crushing strength of aggregates", Journal of the Mechanics and Physics of Solids, 122, pp. 406-417, 2019. https://doi.org/10.1016/j.jmps.2018.09.030

[22] Ma, Y., Huang, H. "DEM analysis of failure mechanisms in the intact Brazilian test", International Journal of Rock Mechanics and Mining Sciences, 102, pp. 109-119, 2018. https://doi.org/10.1016/j.jirmms.2017.11.010

[23] McDowell, G. R., Yue, P., de Bono, J. P. "Micro mechanics of critical states for isotropically overconsolidated sand", Powder Technology, 283, pp. 440-446, 2015.

https://doi.org/10.1016/j.powtec.2015.05.043

[24] Itasca Consulting Group "Particle flow code, Manual", [online] Available at: https://www.itascacg.com/software/PFC

[25] Potyondy, D. O. "Material-modeling support in PFC: Technical memorandum ICG7766-L", Itasca Consulting Group, Minneapolis, MN, USA, 2015. [online] Available at: https://www.itascacg.com/ software/material-modeling-support

[26] Potyondy, D. O., Cundall, P. A. "A bonded-particle model for rock", International Journal of Rock Mechanics and Mining Sciences, 41(8), pp. 1329-1364, 2004.

https://doi.org/10.1016/j.ijrmms.2004.09.011

[27] Wang, P., Gao, N., Ji, K., Stewart, L., Arson, C. "DEM analysis on the role of aggregates on concrete strength", Computers and Geotechnics, 119, Article No. 103290, 2019. https://doi.org/10.1016/j.compgeo.2019.103290

[28] Ben-Nun, O., Einav, I. "The role of self-organization during confined comminution of granular materials", Philosophical Transactions of the Royal Society A: Mathematical, Physical and Engineering, 368, pp. 231-247, 2009.

https://doi.org/10.1098/rsta.2009.0205

[29] Sukumaran, B., Einav, I., Dyskin, A. "Qualitative assessment of the influence of coordination number on crushing strength using DEM", presented at 5th World Congress on Particle Technology, Orlando, FL, USA, April, 23-27, 2006.

[30] Weibull, W. "A Statistical Distribution Function of Wide Applicability", Journal of Applied Mechanics, 18(3), pp. 293-297, 1951.

https://doi.org/10.1115/1.4010337 